\title{
MICRO-CANTILEVER BIOCHEMICAL SENSING BASED ON ROBUST FINITE-HORIZON NON-LINEAR ESTIMATION
}

\author{
Xin Wang * \\ Department of Electrical Engineering \\ and Renewable Energy Engineering \\ Oregon Institute of Technology \\ Klamath Falls, Oregon 97601, USA \\ Email: xin.wang@oit.edu
}

\author{
Isabelle Dufour \\ Univ. Bordeaux \\ IMS UMR 5218, \\ 351 cours de la Liberation \\ 33400 Talence, France \\ Email: dufour@ixl.fr
}

\begin{abstract}
A novel non-linear estimation based micro-cantilever biochemical rheological sensing technique is proposed in this paper. Contrary to classical rheological measurements using microcantilevers, which is either restricted to resonant phenomena, or based on the measurement of fuid properties over a range of vibration frequencies, the proposed measurement technique can be applied to arbitrary available vibration frequency. By applying non-linear estimation technique, the fluid characteristic parameters including density and viscosity, can be estimated and used to quantify fluid properties. The preliminary simulation studies demonstrate that vibrating micro-cantilevers with nonlinear estimation can be used as effective and robust fluid characterization micro-system in liquid environments. The proposed technique is encouraging for the development of a useful microrheometer on a silicon chip for fluid detection application.
\end{abstract}

\footnotetext{
*Address all correspondence to this author.
}

\author{
Edwin E. Yaz \\ Department of Electrical and Computer Engineering \\ College of Engineering \\ Marquette University \\ Milwaukee, Wisconsin 53201, USA \\ Email: edwin.yaz@marquette.edu \\ Susan C. Schneider \\ Department of Electrical and Computer Engineering \\ College of Engineering \\ Marquette University \\ Milwaukee, Wisconsin 53201, USA \\ Email: susan.schneider@marquette.edu
}

\section{NOMENCLATURE}

Denote the notations as shown in Fig.1.

$\begin{array}{lc}w(x, t) & \text { Vertical deflection } \\ A(x) & \text { Cross sectional area } \\ V(x, t) & \text { Shear force } \\ L & \text { Beam length } \\ E I(x) & \text { Flexural (bending) rigidity } \\ E & \text { Young's elastic modulus for the beam } \\ I(x) & \text { Cross-sectional area moment of inertia }\end{array}$

\section{INTRODUCTION}

Micro-cantilevers have recently attracted much attention as promising and highly sensitive approach for minute quantities detection and have wide range of applicability in chemical, biological and medical fields. Moreover, recent technologies developed in the last few years for the fabrication technologies in micro-electromechanical systems (MEMS) and nano-electromechanical systems (NEMS) have made microcantilevers available miniaturized products. 


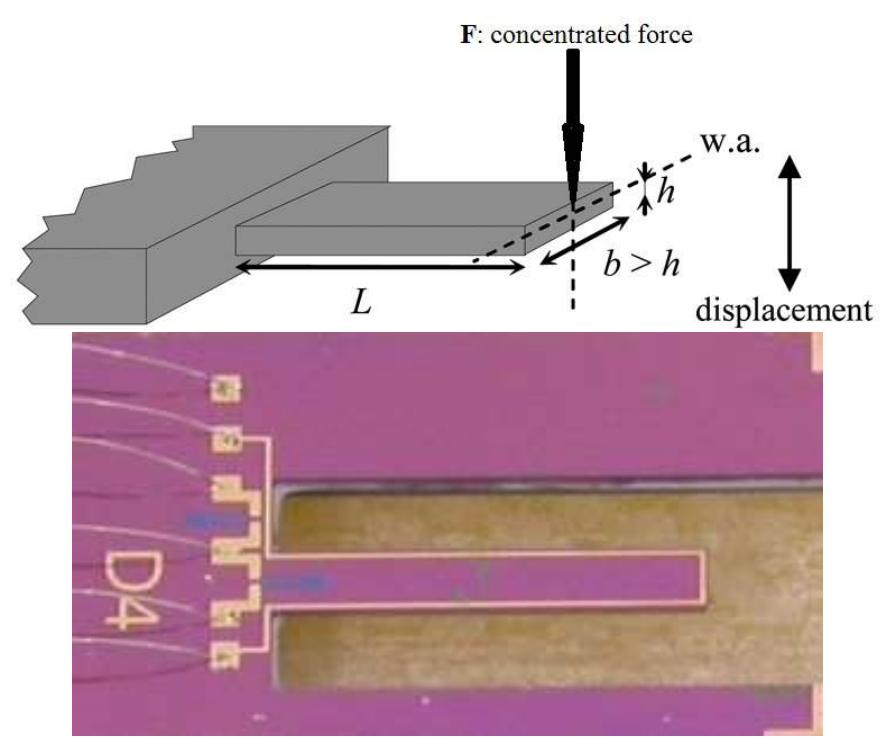

FIGURE 1. MICRO-CANTILEVER SENSING SYSTEM.

Recent literature in micro-cantilevers demonstrates that vibrating micro-cantilevers can be used to quantify fluid properties. Classical rheological measurement using micro-cantilevers is either restricted to resonant phenomena, or based on the measurement of fluid properties over a wide range of frequencies [1-9]. Contrary to the conventional methods, the proposed detection technique can be applied to any excitation vibration frequency. Based on time domain measurement at the arbitrary excitation frequency, non-linear filtering estimation techniques are used to determine the characteristic parameters of the tested liquid.

In the following section, we first derive the mechanical modelling of micro-cantilever system model. The Unscented Kalman Filter (UKF) is developed for rheological fluid properties determination. In the last section, simulation results demonstrate that the proposed non-linear estimation technique can effectively and robustly determine the fluid properties.

The complex response method is used to solve for the partial differential equation. If $w=W \cos (\omega t)$ is a real-valued quantity in the time domain, let us denote $\underline{w}=W e^{j \omega t}$ to represent the corresponding complex notation for $w$, therefore, we have the property of $w=\operatorname{Re}\{\underline{w}\}$.

\section{GENERALIZED MODEL OF RESONANT MICRO- CANTILEVERS IN VISCOUS LIQUID MEDIA}

Before introducing the novel rheological fluid characterization technique with non-linear estimation, the mechanical model is derived in this section. Comparing with classical viscometers or rheometers, micro-cantilever presents some unique property such as larger frequency range measurements and compactness. These unique sensing advantages are due to its mechanical prop- erties $[1,2,11]$. The micro-cantilever rheological sensing system in liquid environment is illustrated as shown in Fig.1. The mechanical system modelling for the micro-cantilever with the concentrated activating force applied at the free-end is derived as follows:

\section{Generalized Equation of Motion}

For transverse bending modes, a micro-cantilever sensor is immersed in fluid. The fixed end of the cantilever is denoted as the origin of the coordinate. The device is activated by the concentrated force applied at the free-end and the movement is affected by distributed force from the liquid.

For mechanics of materials, the beam sustains a bending moment $M(x, t)$, which is related to the beam deflection $w(x, t)$ by

$$
M(x, t)=E I(x) \frac{\partial^{2} w(x, t)}{\partial x^{2}}
$$

Assuming the deflection to be small enough such that the shear deflection is much smaller than $w(x, t)$, i.e. so that the sides of the element $d x$ do not bend, the summation of forces in $y$ direction yields

$$
\left(V(x, t)+\frac{\partial V(x, t)}{\partial x} d x\right)-V(x, t)+f(x, t) d x=\rho A(x) d x \frac{\partial^{2} w(x, t)}{\partial t^{2}}
$$

where

$$
\begin{array}{ll}
V(x, t) & \text { shear force at the left end } \\
\left(V(x, t)+\frac{\partial V(x, t)}{\partial x} d x\right) & \text { shear force at the right end } \\
f(x, t) & \text { fluid force per unit length } \\
\rho A(x) d x \frac{\partial^{2} w(x, t)}{\partial t^{2}} & \text { the inertial force of the element }
\end{array}
$$

Notice that the assumption of small shear deformation used in the force balance of equation (2) is true if $L / h \geq 10, L / b \geq 10$, i.e. for long slender beam, or Euler-Bournoulli beam. $h$ and $b$ are the height and width of the beam, respectively.

Next, moments acting on the element $d x$ about $z$ axis through (Figure 2) point $Q$ are summed, which yields

$$
\begin{array}{r}
\left(M(x, t)+\frac{\partial M(x, t)}{\partial x} d x\right)-M(x, t)+ \\
\left(V(x, t)+\frac{\partial V(x, t)}{\partial x} d x\right) d x+(f(x, t) d x) \frac{d x}{2}=0
\end{array}
$$

where $f(x, t) d x$ is the sum of distributive force. The left hand side of the equation equals zero, since it also assumed that the rotary inertia of the element $d x$ is negligible. 


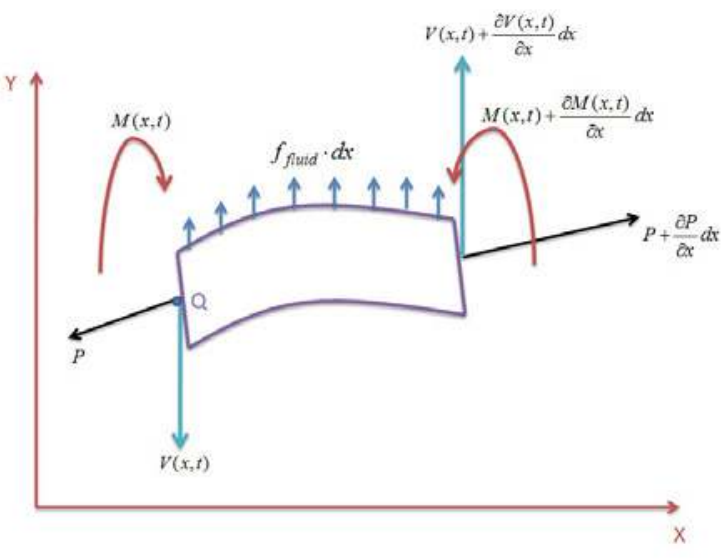

FIGURE 2. FORCE DIAGRAM OF AN INFINITESIMAL ELEMENT OF THE BEAM.

Simplifying the expression yields

$$
\left(\frac{\partial M(x, t)}{\partial x}+V(x, t)\right) d x+\left(\frac{\partial V(x, t)}{\partial x}+\frac{f(x, t)}{2}\right) d x^{2}=0
$$

As $d x \rightarrow 0, d x^{2} \rightarrow 0$ much faster. Therefore, we have

$$
V(x, t)=-\frac{\partial M(x, t)}{\partial x}
$$

This states that the shear force is proportional to the spatial change in the bending moment.

Substitution of this expression for the shear force into Eqn.(2) yields

$$
-\frac{\partial^{2} M(x, t)}{\partial x^{2}} d x+f(x, t) d x=\rho A(x) d x \frac{\partial^{2} w(x, t)}{\partial t^{2}}
$$

Further substitution of the Eqn.(1) into Eqn.(6), and dividing by $d x$ yields:

$$
\rho A(x) \frac{\partial^{2} w(x, t)}{\partial t^{2}}+\frac{\partial^{2}}{\partial x^{2}}\left(E I(x) \frac{\partial^{2} w(x, t)}{\partial x^{2}}\right)=f(x, t)
$$

Consider the effect of the concentrated force acting at the point $x=L$, then Eqn.(7) can be modified to

$$
\rho A(x) \frac{\partial^{2} w(x, t)}{\partial t^{2}}+\frac{\partial^{2}}{\partial x^{2}}\left(E I(x) \frac{\partial^{2} w(x, t)}{\partial x^{2}}\right)=f(x, t)+F \delta(x-L)
$$

Denote $m_{L}=\rho A(x), f=f_{\text {fluid }}$, assuming $E I$ is not a function of $x$, then

$$
m_{L} \frac{\partial^{2} w}{\partial t^{2}}+E I \frac{\partial^{4} w(x, t)}{\partial x^{4}}=f_{\text {fluid }}+F \delta(x-L)
$$

Under the incompressible and Newtonian assumption on the fluid, viscosity and density are intrinsic constants. When a micro-cantilever vibrates in a viscous fluid, the fluid offers resistance to the motion of the beam.

The fluid loading can be interpreted as the sum of two forces: an inertial force, which is proportional to the cantilever acceleration, and a viscous force, which is proportional to the cantilever velocity. Therefore, a viscous fluid exerts on the beam surface the drag force that can be expressed as

$$
f_{\text {fluid }}=-g_{1}(f) \frac{\partial w(t)}{\partial t}-g_{2}(f) \frac{\partial^{2} w(t)}{\partial t^{2}}
$$

From Eqn.(9),(10), we have

$$
E I \frac{\partial^{4} w(x, t)}{\partial x^{4}}+g_{1}(f) \frac{\partial w(t)}{\partial t}+\left(g_{2}(f)+m_{L}\right) \frac{\partial^{2} w}{\partial t^{2}}=F \delta(x-L)
$$

Hence, we have derived the system equation. Now suppose using sinusoidal excitation and adopt complex notations with time dependence $e^{j \omega t}$, we have

$$
E I \frac{\partial^{4} \underline{w}}{\partial x^{4}}+g_{1} j \omega \underline{w}-\left(g_{2}+m_{L}\right) \omega^{2} \underline{w}=0
$$

Equivalently, we have

$$
E I\left(\frac{\partial^{4} \underline{w}}{\partial x^{4}}-\Lambda^{4} \underline{w}\right)=0
$$

where

$$
\Lambda^{4}=\frac{m_{L} \omega^{2}}{E I}\left[1+\frac{g_{2}}{m_{L}}-j \frac{g_{1}}{m_{L} \omega}\right]
$$

Let us consider the boundary conditions $\begin{array}{ll}\left.\frac{w}{\partial w}\right|_{x=0}=0 & \text { no deflection at the fixed end } \\ \left.\frac{\partial w}{\partial x}\right|_{x=0}=0 & \text { no slope at the fixed end }\end{array}$ $\left.E I\left(\frac{\partial^{2} w}{\partial x^{2}}\right)\right|_{x=L}=0 \quad$ no bending moment at the free-end $\left.\frac{\partial}{\partial x}\left[E I \frac{\partial^{2} w}{\partial x^{2}}\right]\right|_{x=L}=\underline{F} \quad$ concentrated shear force at the free-end

The last two conditions are because: if a beam in transverse vibration is free at one end, the deflection and slope $\frac{\partial w}{\partial x}$ at that 
end are not restricted, the bending moment must be zero. Since we have force $F$ acting at the free-end, then shear force is not zero but $F$.

Therefore, we have the following boundary conditions: $\left.\underline{w}\right|_{x=0}=0 \quad$ no deflection at the fixed end

$\left.\frac{\partial w}{\partial x}\right|_{x=0}=0 \quad$ no slope at the fixed end

$\left.\frac{\partial^{2} w}{\partial x^{2}}\right|_{x=L}=0 \quad$ no bending moment at the free-end

$\left.\frac{\partial^{3} w}{\partial x^{3}}\right|_{x=L}=\frac{F}{E I} \quad$ concentrated shear force at the free-end

In this way, we include the effect of $F$ by solving the boundary conditions. Solving the differential Eqn. (13)

$$
E I\left(\frac{\partial^{4} \underline{w}}{\partial x^{4}}-\Lambda^{4} \underline{w}\right)=0
$$

The characteristic equation is

$$
\lambda^{4}-\Lambda^{4}=0
$$

Therefore, we have

$$
\lambda=\Lambda e^{j \frac{2 \pi}{4} n}, \text { for } n=0,1,2,3
$$

Therefore, we have the general solution

$$
\begin{array}{r}
\quad \underline{w}=C_{1} \cos (\Lambda x)+C_{2} \sin (\Lambda x)+C_{3} \cosh (\Lambda x)+C_{4} \sinh (\Lambda x) \\
=C_{1} \cos (\Lambda x)+C_{2} \sin (\Lambda x)+C_{3} \cos (j \Lambda x)+C_{4}(-j \sin (j \Lambda x))
\end{array}
$$
we have

Applying boundary conditions to solve for the coefficients,

$$
-\frac{\cos (\Lambda L) e^{-\Lambda L}+\sin (\Lambda L) e^{-\Lambda L}-\cos (\Lambda L) e^{\Lambda L}+\sin (\Lambda L) e^{\Lambda L}}{E I \Lambda^{3}\left[2+\cos (\Lambda L)\left(e^{\Lambda L}+e^{-\Lambda L}\right)\right]} \underline{F}
$$

\section{MICRO-CANTILEVER SENSING BASED ON NON- LINEAR ESTIMATION}

First, the $g_{1}, g_{2}$ parameters are estimated with the Unscented Kalman Filter. Then, the important micro-cantilever sensing parameters: density and viscosity can be obtained with Maali's equation [10], which can be used to identify the fluid.

\section{UKF Estimation for $g_{1}, g_{2}$ Parameters}

In this section, we set up the Unscented Kalman Filter to estimate the parameters $g_{1}, g_{2}$ by the following system and measurement equations [12]

Denote intermediate parameters

$$
\begin{array}{r}
p_{1}=\frac{g_{2}}{m_{L}} \\
p_{2}=\frac{g_{1}}{m_{L} \omega}
\end{array}
$$

From Eqn.(14), we have

$$
\begin{array}{r}
\Lambda=\sqrt[4]{\frac{m_{L} \omega^{2}}{E I}\left[1+\frac{g_{2}}{m_{L}}-j \frac{g_{1}}{m_{L} \omega}\right]} \\
=\sqrt[4]{\frac{m_{L} \omega^{2}}{E I}\left[1+p_{1}-j p_{2}\right]}
\end{array}
$$

For a given cantilever geometry, for a given viscous fluid at a fixed frequency $g_{1}$ and $g_{2}$ are constant, therefore, the parameters $p_{1}$ and $p_{2}$ are constants. Therefore, we have process equation

$$
\left[\begin{array}{l}
p_{1, k+1} \\
p_{2, k+1}
\end{array}\right]=\left[\begin{array}{ll}
1 & 0 \\
0 & 1
\end{array}\right] \times\left[\begin{array}{l}
p_{1, k} \\
p_{2, k}
\end{array}\right]
$$

The measurement equations are given as follows: From Eqn.(14), we have

$$
\Lambda_{k}=\sqrt[4]{\frac{m_{L} \omega^{2}}{E I}\left[1+p_{1, k}-j p_{2, k}\right]}
$$

Denote the following notation

$$
\begin{array}{r}
\Xi_{k}=\cos \left(\Lambda_{k} L\right) e^{-\Lambda_{k} L}+\sin \left(\Lambda_{k} L\right) e^{-\Lambda_{k} L} \\
-\cos \left(\Lambda_{k} L\right) e^{\Lambda_{k} L}+\sin \left(\Lambda_{k} L\right) e^{\Lambda_{k} L}
\end{array}
$$

Applying boundary conditions (16) to solve coefficients, we have

$$
\begin{array}{r}
w_{k}(x=L)= \\
-\operatorname{Re}\left\{\frac{\Xi_{k}}{E I \Lambda_{k}^{3}\left[2+\cos \left(\Lambda_{k} L\right)\left(e^{\Lambda_{k} L}+e^{-\Lambda_{k} L}\right)\right]} F e^{j \omega t}\right\}+v_{k}
\end{array}
$$

where $v_{k}$ is the measurement noise, assume to be additive white Gaussian noise $v_{k} \sim \mathscr{N}(0, V)$. 
Based on the system and measurement equations above, we set up the following Unscented Kalman Filter for parameters estimation.

\section{System model:}

The new state vector is

$$
\chi_{k}=\left[\begin{array}{l}
p_{1, k} \\
p_{2, k}
\end{array}\right]
$$

with initial condition:

$$
\chi_{0}=\left[\begin{array}{l}
p_{1,0} \\
p_{2,0}
\end{array}\right]
$$

the total number of state variables $n$ equals $2, \kappa$ equals 1 , satisfying the condition $n+\kappa=3$.

\section{Propagating from time step $k$ to $(k+1)$ :}

In the time update part, the 2-dimentional $(n=2) \chi_{k}$ with mean $\hat{\chi}_{k}^{+}$and covariance $P_{k}^{+}$is approximated by $(2 \mathrm{n}+1)$ weighted samples (sigma points) selected by $(2 \mathrm{n}+1)$ weighted samples (sigma points) selected by

$$
\begin{array}{r}
\chi_{k}^{(0)}=\hat{\chi}_{k}^{+} \\
\chi_{k}^{(i)}=\hat{\chi}_{k}^{+}+\tilde{\chi}^{(i)}, i=1,2, \ldots, 2 n \\
\tilde{\chi}^{(i)}=\left(\sqrt{(n+\kappa) P_{k}^{+}}\right), i=1,2, \ldots, n \\
\tilde{\chi}^{(i)}=-\left(\sqrt{(n+\kappa) P_{k}^{+}}\right), i=n+1, n+2, \ldots, 2 n
\end{array}
$$

$\left(\sqrt{(n+\kappa) P_{k}^{+}}\right)$is the $i^{t h}$ row or column of the matrix square root of $(n+\kappa) P_{k}^{+}$, and the $(2 n+1)$ weighting coefficients are given by

$$
\begin{array}{r}
W^{(0))}=\frac{\kappa}{n+\kappa} \\
W^{(i))}=\frac{1}{2(n+\kappa)}, i=1,2, \ldots, 2 n
\end{array}
$$

Each sigma point is initiated through the process model. Since the two parameters are constants, we have

$$
\chi_{k+1}^{(i)}=\chi_{k}^{(i)}=\left[\begin{array}{l}
\chi_{k}^{(i)}(1) \\
\chi_{k}^{(i)}(2)
\end{array}\right]=\left[\begin{array}{l}
p_{1, k}^{(i)} \\
p_{2, k}^{(i)}
\end{array}\right]
$$

The predicted mean is computed by

$$
\hat{\chi}_{k+1}^{-}=\sum_{i=0}^{2 n} W^{(i)} \chi_{k+1}^{(i)}=\frac{1}{n+\kappa}\left\{\kappa \chi_{k+1}^{(0)}+\frac{1}{2} \sum_{i=1}^{2 n} \chi_{k+1}^{(i)}\right\}
$$

The predicted covariance, the priori error covariance, is computed by

$$
\begin{array}{r}
P_{k+1}^{-}=\sum_{i=0}^{2 n} W^{(i)}\left(\chi_{k+1}^{(i)}-\hat{\chi}_{k+1}^{-}\right)\left(\chi_{k+1}^{(i)}-\hat{\chi}_{k+1}^{-}\right)^{T} \\
=\frac{1}{n+\kappa} \times\left\{\begin{array}{c}
\kappa\left(\left(\chi_{k+1}^{(0)}-\hat{\chi}_{k+1}^{-}\right)\left(\chi_{k+1}^{(0)}-\hat{\chi}_{k+1}^{-}\right)^{T}\right)+ \\
\frac{1}{2} \sum_{i=1}^{2 n}\left(\chi_{k+1}^{(i)}-\hat{\chi}_{k+1}^{-}\right)\left(\chi_{k+1}^{(i)}-\hat{\chi}_{k+1}^{-}\right)^{T}
\end{array}\right\}
\end{array}
$$

We implement the measurement update equations as follows. Initialize each predicted observation point using the given observation model. The state variables are the sigma points from the time update part shown above.

$$
\begin{array}{r}
\Lambda_{k+1}^{(i)}=\sqrt[4]{\frac{m_{L} \omega^{2}}{E I}\left[1+p_{1, k+1}^{(i)}-j p_{2, k+1}^{(i)}\right]} \\
=\sqrt[4]{\frac{m_{L} \omega^{2}}{E I}\left[1+\chi_{k+1}^{(i)}(1)-j \chi_{k+1}^{(i)}(2)\right]}
\end{array}
$$

Therefore, we have

$$
\begin{aligned}
\Xi_{k+1}= & \cos \left(\Lambda_{k+1}^{(i)} L\right) e^{-\Lambda_{k+1}^{(i)} L}+\sin \left(\Lambda_{k+1}^{(i)} L\right) e^{-\Lambda_{k+1}^{(i)} L} \\
& -\cos \left(\Lambda_{k+1}^{(i)} L\right) e^{\Lambda_{k+1}^{(i)} L}+\sin \left(\Lambda_{k+1}^{(i)} L\right) e^{\Lambda_{k+1}^{(i)} L}
\end{aligned}
$$

Also, we have

$$
\begin{array}{r}
Y_{k+1}^{(i)}= \\
-\operatorname{Re}\left\{\frac{\Xi_{k+1}}{E I\left(\Lambda_{k+1}^{(i)}\right)^{3}\left[2+\cos \left(\Lambda_{k+1}^{(i)} L\right)\left(e^{\Lambda_{k+1}^{(i)} L}+e^{-\Lambda_{k+1}^{(i)} L}\right)\right]} F e^{j \omega t}\right\}
\end{array}
$$

The predicted observation is calculated by:

$$
\hat{y}_{k+1}=\sum_{i=0}^{2 n} W^{(i)} Y_{k+1}^{(i)}=\frac{1}{n+\kappa}\left\{\kappa Y_{k+1}^{(0)}+\frac{1}{2} \sum_{i=1}^{2 n} Y_{k+1}^{(i)}\right\}
$$


Since the measurement noise is AWGN with covariance V, the covariance of the predicted measurement is:

$$
\begin{array}{r}
P_{y}=V+\sum_{i=0}^{2 n} W^{(i)}\left(Y_{k+1}^{(i)}-y_{k+1}\right)\left(Y_{k+1}^{(i)}-y_{k+1}\right)^{T} \\
=V+\frac{1}{n+\kappa} \times\left\{\begin{array}{c}
\kappa\left(\left(Y_{k+1}^{(0)}-y_{k+1}\right)\left(Y_{k+1}^{(0)}-y_{k+1}\right)^{T}\right)+ \\
\frac{1}{2} \sum_{i=1}^{2 n}\left(Y_{k+1}^{(i)}-y_{k+1}\right)\left(Y_{k+1}^{(i)}-y_{k+1}\right)^{T}
\end{array}\right\}
\end{array}
$$

The cross-correlation is determined by:

$$
\begin{array}{r}
P_{x y}=\sum_{i=0}^{2 n} W^{(i)}\left(\chi_{k+1}^{(i)}-\hat{\chi}_{k+1}^{-}\right)\left(Y_{k+1}^{(i)}-y_{k+1}\right)^{T} \\
=\frac{1}{n+\kappa} \times\left\{\begin{array}{c}
\kappa\left(\left(\chi_{k+1}^{(0)}-\hat{\chi}_{k+1}^{-}\right)\left(Y_{k+1}^{(0)}-y_{k+1}\right)^{T}\right)+ \\
\frac{1}{2} \sum_{i=1}^{2 n}\left(\chi_{k+1}^{(i)}-\hat{\chi}_{k+1}^{-}\right)\left(Y_{k+1}^{(i)}-y_{k+1}\right)^{T}
\end{array}\right\}
\end{array}
$$

The measurement update of the state can be performed by normal Kalman Filter equations

$$
\begin{array}{r}
K_{k+1}=P_{x y} P_{y}^{-1} \\
\hat{\chi}_{k+1}^{+}=\hat{\chi}_{k+1}^{-}+K_{k+1}\left(y_{k+1}-\hat{y}_{k+1}\right) \\
P_{k+1}^{+}=P_{k+1}^{-}-K_{k+1} P_{y} K_{k+1}^{T}
\end{array}
$$

It should be noticed that we have modified the original UKF program in Eqn.(38),(39), by replacing the original $\hat{y}(k+1)$ with $y(k+1)$. Simulation results show that by make this change, the performance of UKF significantly improved.

\section{Viscosity and Density Identification}

Based on the estimated results of the parameters $g_{1}, g_{2}$, the viscosity and density can be identified with Maali's equation [3]:

$$
\begin{array}{r}
\rho=\frac{2 \pi f g_{2}\left(2 a_{1} b_{2}-a_{2} b_{1}\right)+a_{2} \Delta}{\pi^{2} b^{2} f a_{1}\left(a_{1} b_{2}-a_{2} b_{1}\right)} \\
\eta=\frac{a_{1}\left(2 \pi f g_{2} b_{1}+\Delta\right)^{2}}{\pi\left(a_{1} b_{2}-a_{2} b_{1}\right)\left(2 \pi f g_{2}\left(2 a_{1} b_{2}-a_{2} b_{1}\right)+a_{2} \Delta\right)}
\end{array}
$$

where

$$
\Delta=g_{1} a_{2}-\sqrt{\left(2 \pi f g_{2} b_{1}-a_{2} g_{1}\right)^{2}+8 \pi f g_{2} g_{1} a_{1} b_{2}}
$$

TABLE 1. PHYSICAL PARAMETERS

\begin{tabular}{cl}
\hline \multicolumn{1}{c}{ Items } & Values \\
\hline Excitation frequency & $50 \mathrm{~Hz}$ \\
Sampling frequency & $1000 \mathrm{~Hz}$ \\
Size of micro-cantilever & $100 \mu \mathrm{m} \times 20 \mu \mathrm{m} \times 2 \mu \mathrm{m}$ \\
Density of the beam & $2330 \mathrm{~kg} / \mathrm{m}^{3}$ \\
Young's elastic modulus & $169 \mathrm{GPa}$ \\
Quality factor & 1.681 \\
Resonant frequency & $2.425 \mathrm{kHz}$ \\
Amplitude of the excitation force & $1 \mu \mathrm{N}$ \\
\hline
\end{tabular}

TABLE 2. SIMULATION VALUES

\begin{tabular}{c|l|l|l|l}
\hline Items & $g_{1}$ & $g_{2}$ & $\rho$ & $\eta$ \\
\hline Real Values & 1 & $1 \times 10^{-4}$ & $1.931 \times 10^{3}$ & 0.225 \\
Estimated Values & 1.029 & $1.071 \times 10^{-4}$ & $2.153 \times 10^{3}$ & 0.232 \\
\hline
\end{tabular}

The Maali's parameters $a_{1}=1.0553, a_{2}=3.7997, b_{1}=$ $3.8018, b_{2}=2.7364$, and $b$ is the micro-cantilever width. Therefore, based on the estimated parameters $g_{1}, g_{2}$, both of the density $\rho$ and viscosity $\eta$ can be identified.

\section{SIMULATION RESULTS AND ANALYSIS}

The following physical parameters values used in our simulation are summarized in Tab.1.

Without considering the effect of measurement noise, the simulation results are shown below:

It should be noted the percentage error results can be even further improved if more experimental measurement data is taken into account. Simulation results are given in Fig.3 and Fig.4, show that the free-end deflection estimation matches very well with the measured values. Based on 5 seconds of the measurement of the deflection at the free-end, the percentage error for $g_{1}$ is less than about 8 percent, while the percentage error for $g_{2}$ is less than about 3 percent. Therefore, the new method works quite accurately for identifying the liquid properties.

\section{CONCLUSIONS}

In this paper, a new mechanical model for micro-cantilever rheological sensor in liquid environment has been derived and a novel measurement technique based on non-linear estimation technique has been proposed which is significantly different from the classical detection techniques, since it is based on time domain measurement. Simulation results show that this novel tech- 


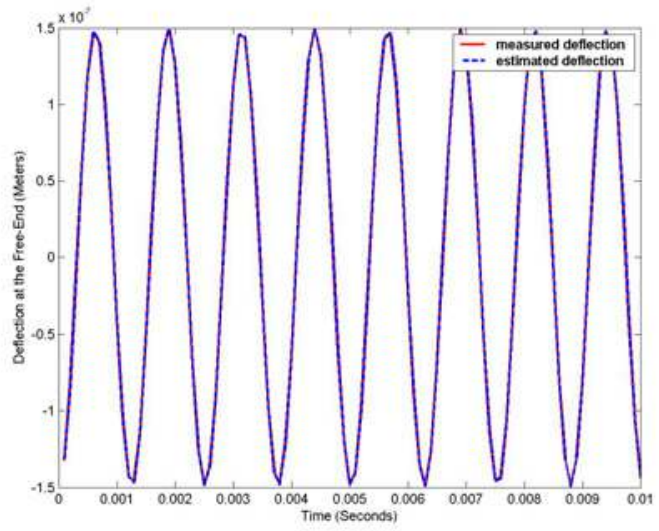

FIGURE 3. DEFLECTION MEASUREMENT AND ESTIMATION AT THE FREE-END.

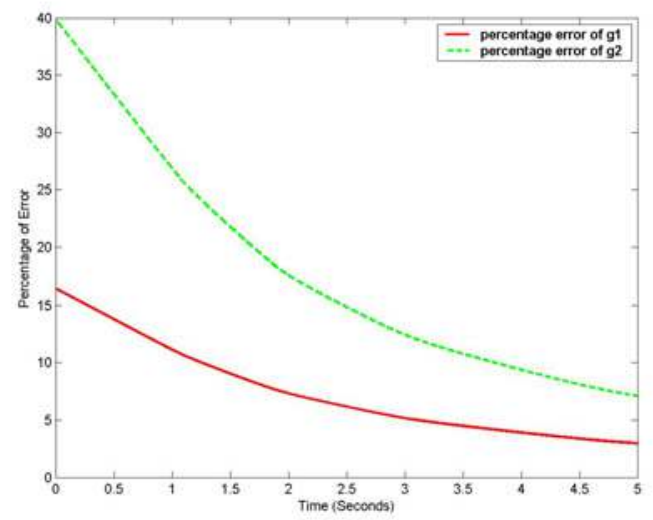

FIGURE 4. PERCENTAGE ERROR OF THE CHARACTERISTIC PARAMETERS.

nique provide accurate estimation performance of the characteristic parameters of the liquid. Therefore, this novel technique can be considered for the next generation biochemical microcantilever sensor applications.

\section{REFERENCES}

[1] Belmiloud N, Dufour I, Colin A, Nicu L (2008) Rheological behaviour probed by vibrating micro-cantilevers, J. Appl. Phys., Vol. 92, 041907.

[2] Cox R, Josse F, Wenzel M. J, Heinrich S. M, Dufour I (2008) Generalized model of resonant polymer-coated micro-cantilevers in viscous liquid media, Anal. Chem., 80, pp. 57605767.

[3] Youssry M, Belmiloud N, Caillard B, Ayela C, Pellet C,
Dufour I (2011) A straightforward determination of fluid viscosity and density using microcantilevers: from experimental data to analytical expressions, Sensors and Actuators A, vol. 172, pp. 40-46.

[4] Dufour I, Fadel L (2003) Resonant microcantilever type chemical sensors: analytical modelling in view of optimization, Sensors and Actuators B, Vol. 91, pp. 353361.

[5] Castille C, Dufour I, Lucat C (2010) Longitudinal vibration mode of piezoelectric thick-film cantilever-based sensors in liquid media, Appl. Phys. Lett., Vol. 96, 154102.

[6] Belmiloud N, Lochon F, Dufour I, Guillot P, Guirardel M, Colin A, Nicu L (2006) Determination of fluid viscoelastic properties using resonant micro-cantilever, International Workshop on Nanomechanical Sensors, Copenhagen, Denmark, May 7-10.

[7] Heinrich SM, Wenzel MJ, Josse F, Dufour I(2009) An analytical model for transient deformation of viscoelastically coated beams: applications to static-mode microcantilever chemical sensors, J. Appl. Phys., Vol. 105, 124903.

[8] Dufour I, Lochon F, Heinrich SM, Josse F, Rebire D (2007) Effect of coating viscoelasticity on quality factor and limit of detection of micro-cantilever chemical sensors, IEEE Sensors J., Vol. 7, No. 2.

[9] Dufour I, Heinrich SM, and Josse F (2007), Theoretical analysis of strong-axis bending mode vibrations for resonant micro-cantilever (bio)chemical sensors in gas or liquid phase, IEEE J. of Micro-electromechanical Sys.. Vol. 16, No. 1.

[10] Maali A, Hurth C, Boisgard R, Jai C, Cohen-Bouhacina T, and Aim J. P (2005) J. Appl. Phys., Vol. 97, 074907.

[11] Rosenhead L (1963) Laminar Boundary Layers, Clarendon, Oxford.

[12] Wang X, Yaz E (2009) A New Nonlinear-Filter-Based Modulation/Demodulation Technique for Chaotic Communication, Proc. of 2009 American Control Conf., St Louis, Missouri, pp.1876-1881.

[13] Wang X, Yaz E.E (2009) The State Dependent Control of Continuous-Time Non-linear Systems with Mixed Performance Criteria, Proc. of IASTED Int. Conf. on Identi cation Control and Applications, Honolulu, HI, pp. 98102.

[14] Wang X, Yaz E.E (2010) Robust multi-criteria optimal fuzzy control of continuous-time non-linear systems, Proc. of the 2010 American Control Conference, Baltimore, MD, USA, pp. 6460-6465.

[15] Wang X, Yaz E.E, Jeong C.S (2010) Robust non-linear feedback control of discrete-time non-linear systems with mixed performance criteria, Proc. of the 2010 American Control Conference, Baltimore, MD, USA,pp. 6357-6362.

[16] Wang X, Yaz E.E (2010) Robust multi-criteria optimal fuzzy control of discrete-time non-linear systems, Proc. of 
the 49th IEEE Conference on Decision and Control, Atlanta, Georgia, USA, pp. 4269-4274.

[17] Wang X, Yaz E.E, Yaz Y.I (2010) Robust and resilient state dependent control of continuous-time non-linear systems with general performance criteria, Proc. of the 49th IEEE Conference on Decision and Control, Atlanta, Georgia, USA, pp. 603-608.

[18] Wang X, Yaz E.E, Yaz Y.I, Robust and resilient state dependent control of discrete time non-linear systems with general performance criteria, Proc. of the 18th IFAC Congress, Milano, Italy, pp. 10904-10909. 